\title{
Revising UNITY Programs: Possibilities and Limitations $^{1}$
}

\author{
Ali Ebnenasir, Sandeep S. Kulkarni, and Borzoo Bonakdarpour \\ Software Engineering and Network Systems Laboratory \\ Department of Computer Science and Engineering \\ Michigan State University \\ 48824 East Lansing, Michigan, USA \\ \{ebnenasi, sandeep, borzoo\}@cse.msu.edu \\ http: //www.cse.msu.edu/ \{ebnenasi, sandeep, borzoo\}
}

\begin{abstract}
We concentrate on automatic addition of UNITY properties unless, stable, invariant, and leads-to to programs. We formally define the problem of adding UNITY properties to programs while preserving their existing properties. For cases where one simultaneously adds a single leads-to property along with a conjunction of unless, stable, and invariant properties to an existing program, we present a sound and complete algorithm with polynomial time complexity (in program state space). However, for cases where one simultaneously adds two leads-to properties to a program, we present a somewhat unexpected result that such addition is NP-complete. Therefore, in general, adding one leads-to property is significantly easier than adding two (or more) leads-to properties.
\end{abstract}

Keywords: UNITY, Formal Methods, Program Synthesis

\section{Introduction}

In this paper, we focus on automated addition of UNITY properties [1] to existing programs. To motivate the application of this work, consider two scenarios: In the first scenario, a designer checks the model of a computing system to determine if it satisfies the given properties of interest using a model checker. The model checker provides a counterexample demonstrating that one of the properties is not met. In this scenario, the designer needs to modify the given model so that it satisfies that property (while ensuring that the remaining properties continue to be satisfied). In another scenario, an existing program needs to be modified so that it satisfies an additional property of interest (while satisfying existing properties). Such a scenario occurs when the specification is incomplete and as designers gain more domain knowledge about the problem at hand, they may add new properties to the specification.

\footnotetext{
1 This work was partially sponsored by NSF CAREER CCR-0092724, DARPA Grant OSURS01-C-1901, ONR Grant N00014-01-1-0744, NSF grant EIA-0130724, and a grant from Michigan State University.
} 
There exist two ways in which one can deal with the above scenarios: (1) local redesign, where the designer removes the program behaviors that violate the property of interest without adding any new behaviors, or (2) comprehensive redesign, where the designer introduces new behaviors in the program computations (e.g., by introducing new variables, or adding new computation paths). Clearly, the former approach is desirable, as it ensures that certain existing specifications (e.g., the UNITY specifications from [1]) are preserved. Moreover, in the second scenario, the designer may not have access to the complete specification of the existing system. Hence, in this case, local redesign, if successful, is highly desirable.

We expect that an algorithm for local redesign would be especially useful if it were sound and complete. A sound algorithm ensures that the redesigned program meets the new specification (in addition to preserving existing specification); i.e., the redesigned program is correct by construction. Moreover, a complete algorithm provides an insight for the designer to decide if a program can be redesigned locally or it should be redesigned from scratch to satisfy a new property while preserving its exiting properties. Such automated assistance for the designer is highly desirable since it significantly decreases the design time by warning the designers about spending time on fixing a program that is not fixable.

With this motivation, we present an incremental method for adding UNITY properties to programs. Our incremental approach has the potential to reuse the computations of an existing program while adding new properties to it. Also, we focus on UNITY since it provides (i) a simple and general computational model for a variety of computing systems, and (ii) a proof system for refining programs [1]. We expect to benefit from simplicity and generality of UNITY in automatic design of programs.

The basic UNITY properties from [1] are unless, stable, invariant, ensures, and leads-to. (We refer the reader to Section 2 for precise definitions.) Of these, ensures can be expressed in terms of leads-to and unless. Hence, we focus on adding unless, stable, invariant, and leads-to to programs. In particular, we present a sound and complete algorithm for simultaneous addition of a single leads-to property and a conjunction of unless, stable, and invariant properties. The time complexity of our algorithm is polynomial in program state space. However, we present an unexpected result that simultaneous addition of two leads-to properties to a program is NP-complete. Based on this result, we find that adding one leads-to property is significantly easier than simultaneous addition of two (or more) leads-to properties.

Contributions. The contributions of this paper are as follows: (1) We formally define the problem of adding UNITY properties to programs; (2) We present a sound and complete algorithm for automatic addition of a leads-to property and a conjunction of unless, stable, and invariant properties to programs, and (3) We show that simultaneous addition of two leads-to properties to a program is NP-complete. 
Organization of the paper. First, we present preliminary concepts in Section 2. In Section 3, we formally define the problem of adding UNITY properties to programs. Then, in Section 4, we present our sound and complete algorithm for adding a leads-to property to programs. In Section 5, we present our NPcompleteness result. Subsequently, in Section 6, we demonstrate our addition algorithm using a mutual exclusion program. In Section 7, we compare the results of this paper with related work. We discuss the limitations and the applications of our results in Section 8. Finally, we make concluding remarks in Section 9.

\section{Preliminaries}

In this section, we give formal definitions of programs and properties in UNITY [1]. Programs are defined in terms of their state space and their transitions. UNITY properties are defined in terms of infinite sequences of transitions.

Program. A program $p$ is of the form $\left\langle S_{p}, I_{p}, \delta_{p}\right\rangle$, where $S_{p}$ is a finite set of states, $I_{p} \subseteq S_{p}$ is the set of initial states of $p$, and $\delta_{p} \subseteq S_{p} \times S_{p}$ is the set of transitions of $p$.

A state predicate of $p$ is any subset of $S_{p}$. A sequence of states, $\sigma=\left\langle s_{0}, s_{1}, \cdots\right\rangle$ is a computation of $p$ iff (if and only if) the following three conditions are satisfied: (1) $s_{0} \in I_{p}$; (2) if $\sigma$ is infinite then $\forall j: j>0:\left(s_{j-1}, s_{j}\right) \in \delta_{p}$ holds, and (3) if $\sigma$ is finite and terminates in state $s_{f}$ then there does not exist state $s$ such that $\left(s_{f}, s\right) \in \delta_{p}$, and $\forall j: 0<j \leq f:\left(s_{j-1}, s_{j}\right) \in \delta_{p}$ holds. A sequence of states, $\left\langle s_{0}, s_{1}, \ldots, s_{n}\right\rangle$, is a computation prefix of $p$ iff $\forall j: 0<j \leq n:\left(s_{j-1}, s_{j}\right) \in \delta_{p}$.

Properties of UNITY Programs. We reiterate the definition of the UNITY properties from [1]. In the following definitions, $P$ and $Q$ are state predicates.

- Unless. An infinite sequence of states $\sigma=\left\langle s_{0}, s_{1}, \ldots\right\rangle$ satisfies $P$ unless $Q$ iff $\forall i: 0 \leq i:\left(s_{i} \in(P \cap \neg Q)\right) \Rightarrow\left(s_{i+1} \in(P \cup Q)\right)$. Intuitively, the sequence $\sigma$ satisfies $P$ unless $Q$ iff if $P$ holds in some state of $\sigma$ then either (1) $Q$ never holds in $\sigma$ and $P$ is continuously true, or (2) $Q$ eventually becomes true and $P$ holds at least until $Q$ becomes true.

- Stable. An infinite sequence of states $\sigma=\left\langle s_{0}, s_{1}, \ldots\right\rangle$ satisfies stable $(P)$ iff $\sigma$ satisfies ( $P$ unless false). Intuitively, $P$ is stable iff once it becomes true it remains true forever.

- Invariant. An infinite sequence of states $\sigma=\left\langle s_{0}, s_{1}, \ldots\right\rangle$ satisfies invariant $(P)$ iff $s_{0} \in P$ and $\sigma$ satisfies stable $(P)$. An invariant property always holds.

- Ensure. An infinite sequence of states $\sigma=\left\langle s_{0}, s_{1}, \ldots\right\rangle$ satisfies $P$ ensures $Q$ iff $(\sigma$ satisfies $P$ unless $Q)$ and $\left(\exists j: 0 \leq j: s_{j} \in Q\right)$. In other words, there exists a state $s_{j}$, where (i) $Q$ eventually becomes true in $s_{j}$, and (ii) $P$ remains true everywhere between the first state $s_{i}, i \leq j$, where $P$ becomes true and $s_{j}$.

- Leads-to (denoted $\mapsto)$. An infinite sequence of states $\sigma=\left\langle s_{0}, s_{1}, \ldots\right\rangle$ satisfies $P \mapsto Q$ iff $\left(\forall i: 0 \leq i:\left(s_{i} \in P\right) \Rightarrow\left(\exists j: i \leq j: s_{j} \in Q\right)\right)$. If $P$ holds in some state $s_{i} \in \sigma$ then there exists a state $s_{j} \in \sigma$ where $Q$ holds and $i \leq j$.

Since ensures can be expressed as a conjunction of an unless property and a leads-to property, we do not consider it explicitly in this paper. The properties 
unless, stable, and invariant are safety properties, as defined by Alpern and Schneider [2]. These properties can be modeled in terms of a set of bad transitions that should never occur in a program computation. For example, stable $(P)$, requires that transitions of the form $\left(s_{0}, s_{1}\right)$, where $s_{0} \in P$ and $s_{1} \notin P$ should never occur in any program computation. Hence, for simplicity, in this paper, when dealing with these properties, we assume that they are represented as a set of transitions $\mathcal{B} \subseteq S_{p} \times S_{p}$ that must not occur in any computation.

Now, let spec be any conjunction of the above properties; i.e., spec $=\mathcal{L}_{1} \wedge$ $\cdots \wedge \mathcal{L}_{n}$, where $\mathcal{L}_{i}$ belongs to the set of properties unless, stable, invariant, and leads-to $(1 \leq i \leq n)$. A sequence of states $\sigma=\left\langle s_{0}, s_{1}, \ldots\right\rangle$ satisfies spec iff $\forall i: 1 \leq i \leq n: \sigma$ satisfies $\mathcal{L}_{i}$. We say that program $p$ satisfies a given UNITY specification, spec, iff all computations of $p$ are infinite and every computation of $p$ satisfies spec.

Remark. We distinguish between a terminating computation and a deadlocked computation. To model a computation that terminates in state $s_{f}$, we include the transition $\left(s_{f}, s_{f}\right)$ in program $p$. When a computation $c$ of $p$ reaches $s_{f}, c$ can be extended to an infinite computation by stuttering at $s_{f}$. If there exists a state $s_{d}$ such that there is no outgoing program transition from $s_{d}$ then $s_{d}$ is a deadlocked state and a computation of $p$ that reaches $s_{d}$ is a deadlocked computation. Such computations cannot be extended to an infinite computation. We want to ensure that such deadlocked computations do not occur while revising a program.

\section{Problem Statement}

In this section, we formally define the problem of adding UNITY specifications to programs. Given is a program $p$ (with state space $S_{p}$, initial states $I_{p}$ and transitions $\delta_{p}$ ) that satisfies a UNITY specification $s p e c_{e}$. The goal is to generate a modified version of $p$, denoted $p^{\prime}$, in such a way that $p^{\prime}$ satisfies a UNITY specification $s{ }^{2} c_{n}$, in addition to preserving its existing specification spec $_{e}$. Moreover, this addition should be done in such a way that one does not need to know the existing specification $s e_{e}$; during the addition, we only want to reuse the correctness of $p$ with respect to $\operatorname{spec}_{e}$ so that the correctness of $p^{\prime}$ with respect to spec $_{e}$ is derived from ' $p$ satisfies $s p e c_{e}$ '.

Now, we identify constraints on $S_{p^{\prime}}, I_{p^{\prime}}$ and $\delta_{p^{\prime}}$. Clearly, in obtaining $S_{p^{\prime}}$, no new states should be added to $S_{p}$; otherwise, there is no guarantee that the correctness of $p$ can be reused to ensure that existing specification will continue to be preserved. Moreover, since $S_{p}$ denotes the set of all states (not just reachable states) of $p$, removing states from $S_{p}$ is not advantageous. Likewise, $I_{p^{\prime}}$ should not have any states that were not there in $I_{p}$. Moreover, since $I_{p}$ denotes the set of all initial states of $p$, we should preserve them during the transformation. Finally, likewise, $\delta_{p^{\prime}}$ should be a subset of $\delta_{p}$. Note that not all transitions of $\delta_{p}$ may be preserved in $p^{\prime}$. However, we must ensure that $p^{\prime}$ does not deadlock in any reachable state. Based on the definition of the UNITY specification, if (i) $\delta_{p^{\prime}} \subseteq \delta_{p}$, (ii) $p^{\prime}$ does not deadlock in any reachable state, and (iii) $p$ satisfies spec $_{e}$, then $p^{\prime}$ also satisfies spec $_{e}$. Thus, the problem statement is defined as follows: 
The Problem of Adding UNITY Properties

Given a program $p$, its state space $S_{p}$, its set of initial states $I_{p}$, and

a UNITY specification $\operatorname{spec}_{n}$, identify

$\delta_{p^{\prime}}, S_{p^{\prime}}$, and $I_{p^{\prime}}$ such that

(C1) $\quad S_{p^{\prime}}=S_{p}$

(C2) $\quad I_{p^{\prime}}=I_{p}$

(C3) $\quad \delta_{p^{\prime}} \subseteq \delta_{p}$

(C4) $p^{\prime}$ satisfies spec $_{n}$

Note that the requirement of deadlock freedom is not explicitly specified in the above problem statement, as it follows from ' $p$ ' satisfies spec $_{n}$ '.

\section{Adding Single Leads-to and Multiple Safety Properties}

In this section, we present a simple solution for the addition problem (defined in Section 3) for the case where the new specification $s p e c_{n}$ is a conjunction of a single leads-to property and multiple safety properties. We note that the goal of our algorithm is simply to illustrate the feasibility of this solution. Hence, although our algorithm in this section can be modified to reduce complexity further, we have chosen to present a simple (and not so efficient) solution. In Section 8 , we give an intuition as to how one can implement our algorithm using counterexamples provided by model checkers.

Given are a program $p=\left\langle S_{p}, I_{p}, \delta_{p}\right\rangle$ and a specification spec $_{n}=\mathcal{B} \wedge \mathcal{L}$, where $\mathcal{B}$ represents the conjunction of a set of safety properties and $\mathcal{L}$ is a $R \mapsto T$ property for state predicates $R$ and $T$. Our goal is to generate a new program $p^{\prime}$ that satisfies $s p e c_{n}$ and preserves the existing specification. To guarantee that $p^{\prime}$ satisfies $\mathcal{B}$ (i.e., $p^{\prime}$ never executes a transition in the set of bad transitions $\mathcal{B}$ ), we exclude all transitions of $p$ that belong to $\mathcal{B}$ (see Step 1 in Figure 1). To add the leads-to property $\mathcal{L} \equiv(R \mapsto T)$ to $p$, we need to guarantee that any computation of $p^{\prime}$ that reaches a state in $R$ will eventually reach a state in $T$. Towards this end, we rank all states $s$ based on the length of the shortest computation prefix of $p$ from $s$ to a state in $T$. In such ranking, if no state of $T$ is reachable from $s$ then the rank of $s$ will be infinity. Also, the rank of states in $T$ is zero.

There exist two obstacles in guaranteeing the reachability from $R$ to $T:(1)$ the deadlock states reachable from $R$, and (2) cycles reachable from $R$ where the computations of $p^{\prime}$ may be trapped forever. We may create deadlock states by (i) removing safety-violating transitions (Step 1 in Figure 1), and (ii) making infinity-ranked states unreachable in Step 3.

To deal with the deadlock states, we make them unreachable by removing transitions that reach a deadlock state (Step 4 in Figure 1). Such removal of transitions may introduce new deadlock states that are removed in the while loop in Step 4. If the removal of deadlock states culminates in making an initial state deadlocked then $(R \mapsto T)$ cannot be added to $p$. Otherwise, we again rank all states (in Step 5) since we might have removed some deadlock states in $T$, and as a result, we might have created new infinity-ranked states. We repeat the above steps until no reachable state in $R$ has the rank infinity. At this point (end of repeat-until in Step 6), there is a path from each state in $R$ to a $T$ state. However, there may be cycles that are reachable from a state in $R$. 


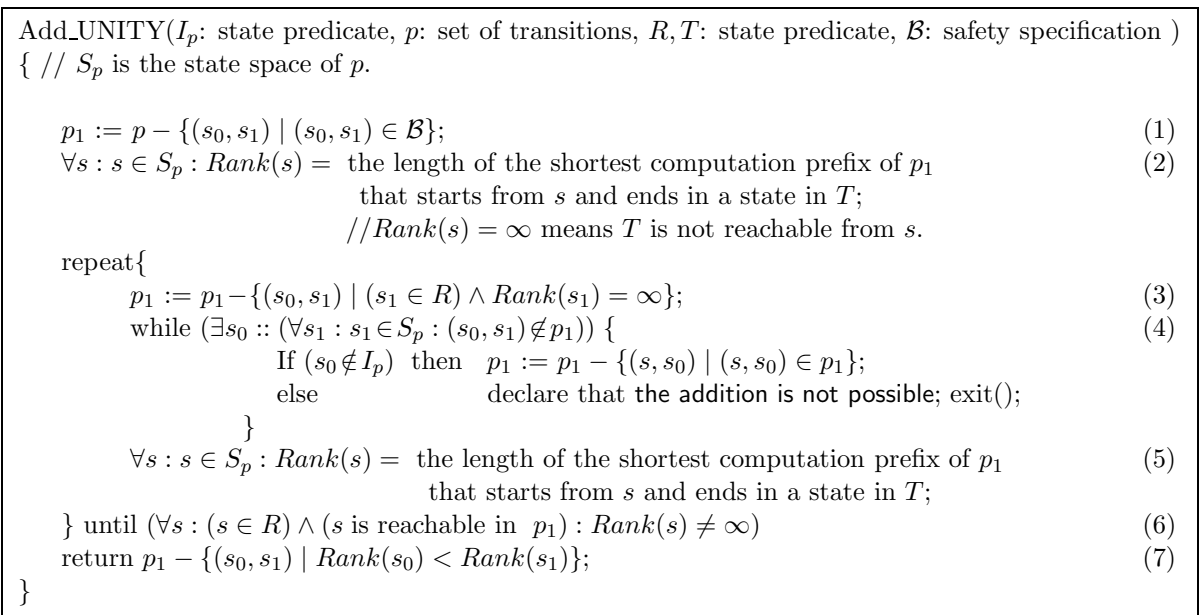

Fig. 1. Adding one leads-to and multiple safety properties.

To deal with such cycles from $R$, we remove transitions from low-ranked states to high-ranked states (Step 7 in Figure 1). In particular, if $\operatorname{Rank}\left(s_{0}\right)<\operatorname{Rank}\left(s_{1}\right)$ then that means there exists a shorter computation prefix from $s_{0}$ to $T$ with respect to the computation prefix from $s_{1}$ to $T$. Thus, removing $\left(s_{0}, s_{1}\right)$ will not make $s_{0}$ deadlocked. (Note that in Step 7, transitions of the form $\left(s_{0}, s_{1}\right)$, where $\operatorname{Rank}\left(s_{0}\right)=\infty$ and $\operatorname{Rank}\left(s_{1}\right)=\infty$, are not removed. Hence, computations in which neither predicates $R$ and $T$ are reached will not be affected. )

Theorem 4.1 The algorithm Add_UNITY is sound.

Proof. Since Add_UNITY does not add any new states to $S_{p}$, we have $S_{p^{\prime}}=$ $S_{p}$. Likewise, Add_UNITY does not remove (respectively, introduce) any initial states; we have $I_{p^{\prime}}=I_{p}$. The Add_UNITY algorithm only updates $\delta_{p}$ by excluding some transitions from $\delta_{p}$ in Steps $1,3,4$, and 7. It follows that $\delta_{p^{\prime}} \subseteq \delta_{p}$. By construction, Add_UNITY removes all deadlock states in Step 4. Thus, if Add_UNITY generates a program $p^{\prime}$ in Step 7 then reachability from $R$ to $T$ is guaranteed in $p^{\prime}$. Thus, $p^{\prime}$ meets all the requirements of the addition problem.

Theorem 4.2 The algorithm Add_UNITY is complete.

Proof. Note that any transition removed in Add_UNITY (in Steps 1, 3, and 4) must be removed in any program that meets the requirements of the addition problem. Hence, when failure is declared (in Step 4), it follows that a solution to the addition problem does not exist.

Theorem 4.3 The time complexity of Add_UNITY algorithm is polynomial in $S_{p}$.

Proof. The proof follows from the polynomial-time complexity of each step of Add_UNITY.

In Section 6, we demonstrate our algorithm in the local redesign of a token passing mutual exclusion program. We have also used our algorithm in the local redesign of a readers-writers program in [3]. 


\section{$5 \quad$ Adding Two Leads-to Properties}

In this section, we show that the addition of a UNITY specification, which is the conjunction of two leads-to properties, to a program is NP-complete. We show this by presenting a reduction from the 3-SAT problem to an instance of the decision problem defined below. The instance and the decision problem for adding two leads-to properties are as follows:

Instance. An instance of the addition problem for two leads-to properties consists of a program $p$, its state space $S_{p}$, set of initial states $I_{p}$, transitions $\delta_{p}$, and spec $_{n}=\mathcal{L}_{1} \wedge \mathcal{L}_{2}$, where $\mathcal{L}_{1} \equiv P \mapsto Q$ and $\mathcal{L}_{2} \equiv R \mapsto T$, and $P, Q, R$, and $T$ are state predicates.

The decision problem.

Given is an instance of the addition problem for two leads-to properties:

Does there exist a program $p^{\prime}$, its state space $S_{p^{\prime}}$, and its set of initial states $I_{p^{\prime}}$ such that

$$
S_{p}=S_{p^{\prime}}, I_{p^{\prime}}=I_{p}, \delta_{p^{\prime}} \subseteq \delta_{p} \text {, and } p^{\prime} \text { satisfies spec }{ }_{n}=\mathcal{L}_{1} \wedge \mathcal{L}_{2} \text { ? }
$$

The 3-SAT problem is as follows: Let $x_{1}, x_{2}, \ldots, x_{n}$ be propositional variables. Given is a Boolean formula $y=y_{1} \wedge y_{2} \cdots \wedge y_{M}$, where each $y_{j}(1 \leq j \leq M)$ is a disjunction of exactly three literals. Does there exist an assignment of truth values to $x_{1}, x_{2}, \ldots, x_{n}$ such that $y$ is satisfiable?

Next, in Subsection 5.1, we present a polynomial-time mapping from 3-SAT to an instance of the decision problem. Then, in Subsection 5.2, we show that the 3-SAT problem is satisfiable iff the answer to the above decision problem is affirmative for the instance introduced in Subsection 5.1.

\subsection{Mapping 3-SAT to the Addition of Two Leads-to Properties}

We now present the mapping of an instance of the 3-SAT problem to an instance of the problem of adding two leads-to properties. First, we introduce the state space and the initial states of the instance of the addition problem corresponding to each variable $x_{i}$ and each disjunction $y_{j}$. We also introduce the state predicates $P, Q, R$, and $T$ that define $\operatorname{spec}_{n}$. Then, we present the transitions of the instance corresponding to each variable $x_{i}$ and each disjunction $y_{j}$.

The state space, initial states, and state predicates $P, Q, R$, and $T$. Corresponding to each variable $x_{i}$ of the given 3-SAT instance, we introduce six states $P_{i}, a_{i}, Q_{i}, R_{i}, b_{i}$, and $T_{i}$, where $1 \leq i \leq n$ (see Figure 2). For each disjunction $y_{j}$, we introduce a state $c_{j}$, where $1 \leq j \leq M$, in the state space. Thus,

$$
\begin{aligned}
& -S_{p}=\left\{P_{i}, a_{i}, Q_{i}, R_{i}, b_{i}, T_{i} \mid 1 \leq i \leq n\right\} \cup\left\{c_{j} \mid 1 \leq j \leq M\right\} \\
& -I_{p}=\left\{c_{j} \mid 1 \leq j \leq M\right\} \\
& -P=\left\{P_{i} \mid 1 \leq i \leq n\right\}, Q=\left\{Q_{i} \mid 1 \leq i \leq n\right\}, R=\left\{R_{i} \mid 1 \leq i \leq n\right\}, \text { and } \\
& \quad T=\left\{T_{i} \mid 1 \leq i \leq n\right\}
\end{aligned}
$$

The program transitions. Corresponding to each variable $x_{i}$, we include transitions $\left(P_{i}, a_{i}\right),\left(a_{i}, b_{i}\right),\left(b_{i}, Q_{i}\right),\left(Q_{i}, Q_{i}\right),\left(R_{i}, b_{i}\right),\left(b_{i}, a_{i}\right),\left(a_{i}, T_{i}\right)$, and $\left(T_{i}, T_{i}\right)$ in the set of program transitions $\delta_{p}$ (see Figure 2). Moreover, corresponding to each disjunction $y_{j}$, we include the following transitions: 


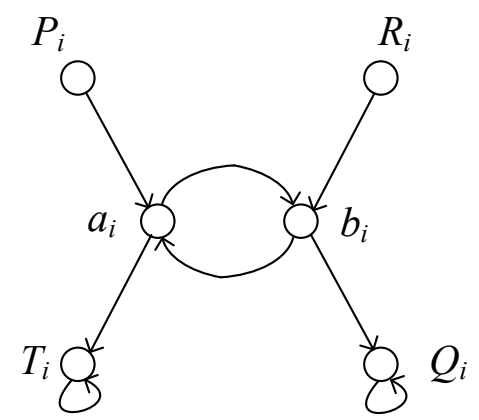

Fig. 2. Mapping of variables in the 3-SAT problem.

- If $x_{i}$ is a literal in $y_{j}$ then we include the transition $\left(c_{j}, P_{i}\right)$.

- If $\neg x_{i}$ is a literal in $y_{j}$ then we include the transition $\left(c_{j}, R_{i}\right)$.

\subsection{Reduction from the 3-SAT Problem}

In this subsection, we show that the given instance of 3-SAT is satisfiable iff both leads-to properties $\mathcal{L}_{1} \equiv(P \mapsto Q)$ and $\mathcal{L}_{2} \equiv(R \mapsto T)$ can be added to the problem instance defined in Subsection 5.1.

Part I. First, we show that if the given instance of the 3-SAT formula is satisfiable then there exists a solution that meets the requirements of the decision problem. Since the 3-SAT formula is satisfiable, there exists an assignment of truth values to variables $x_{i}, 1 \leq i \leq n$, so that each $y_{j}, 1 \leq j \leq M$, is true. Now, we identify a program $p^{\prime}$ that is obtained by adding the leads-to properties $\mathcal{L}_{1}$ and $\mathcal{L}_{2}$ to program $p$ as follows.

- The state space of $p^{\prime}$ consists of all the states of $p$, i.e., $S_{p^{\prime}}=S_{p}$.

- The initial states of $p^{\prime}$ consists of all the initial states of $p$, i.e., $I_{p^{\prime}}=I_{p}$.

- For each variable $x_{i}$, if $x_{i}$ is true then we include the transitions $\left(P_{i}, a_{i}\right)$, $\left(a_{i}, b_{i}\right),\left(b_{i}, Q_{i}\right)$, and $\left(Q_{i}, Q_{i}\right)$.

- For each variable $x_{i}$, if $x_{i}$ is false then we include the transitions $\left(R_{i}, b_{i}\right)$, $\left(b_{i}, a_{i}\right),\left(a_{i}, T_{i}\right)$, and $\left(T_{i}, T_{i}\right)$.

- For each disjunction $y_{j}$ that contains $x_{i}$, we include the transition $\left(c_{j}, P_{i}\right)$ if $x_{i}$ is true.

- For each disjunction $y_{j}$ that contains $\neg x_{i}$, we include the transition $\left(c_{j}, R_{i}\right)$ if $x_{i}$ is false.

As an illustration, we show the partial structure of $p^{\prime}$, for the formula $\left[\left(x_{1} \vee\right.\right.$ $\left.\left.\neg x_{2} \vee x_{3}\right) \wedge\left(x_{1} \vee x_{2} \vee \neg x_{4}\right)\right]$, where $x_{1}=$ true, $x_{2}=$ false, $x_{3}=$ false, and $x_{4}=$ false in Figure 3 .

Now, we show that $p^{\prime}$ meets the requirements of the decision problem.

- The first three constraints of the decision problem are trivially satisfied. 


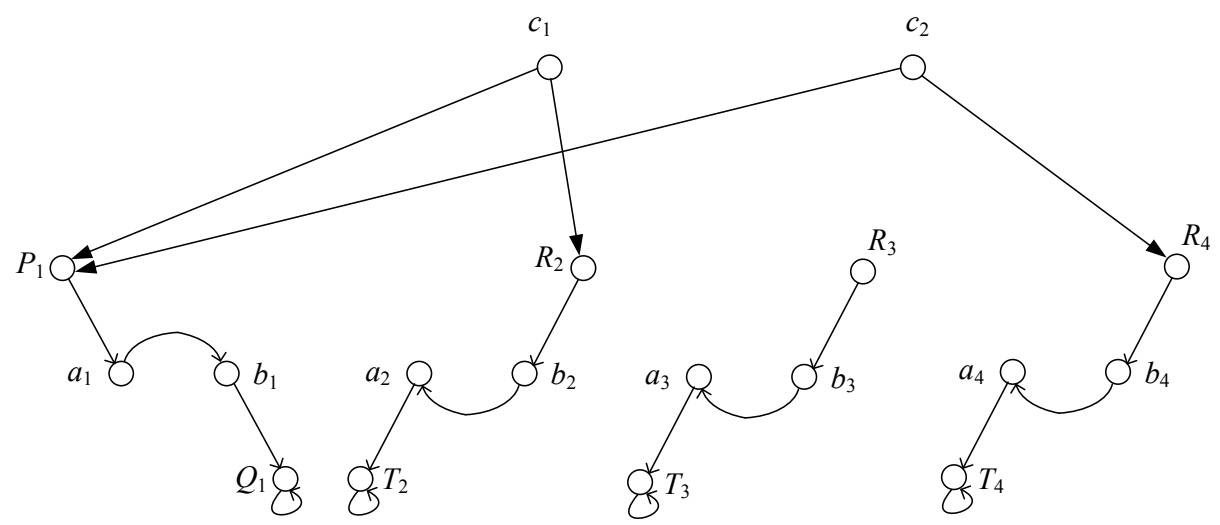

Fig. 3. The partial structure of the revised program.

- It is easy to observe that by construction, there are no deadlock states. Hence, for the UNITY specification $\operatorname{spec}_{e}$ if $p$ satisfies $\operatorname{spec}_{e}$ then $p^{\prime}$ also satisfies $s p e c_{e}$. Moreover, if a computation of $p^{\prime}$ reaches $P_{i}$ from some initial state (i.e., $x_{i}$ is true) then that computation will eventually reach $Q_{i}$ and will stay there, since $p^{\prime}$ does not include the transition $\left(b_{i}, a_{i}\right)$. Likewise, if a computation of $p^{\prime}$ reaches $R_{i}$ from some initial state (i.e., $x_{i}$ is false) then that computation will eventually reach $T_{i}$ and will stay there, since $p^{\prime}$ does not include the transition $\left(a_{i}, b_{i}\right)$. Thus, $p^{\prime}$ satisfies both $\mathcal{L}_{1}$ and $\mathcal{L}_{2}$.

Part II. Next, we show that if there exists a solution to the instance identified in Subsection 5.1, then the given 3-SAT formula is satisfiable. Let $p^{\prime}$ be the program that is obtained by adding the two leads-to properties to program $p$. Now, to obtain the solution for 3-SAT, we proceed as follows. If there exists a computation of $p^{\prime}$ where state $P_{i}$ is reachable then we assign $x_{i}$ the truth value true. Otherwise, we assign it the truth value false.

We now show that the above truth assignment satisfies all disjunctions. Let $y_{j}$ be any disjunction and let $c_{j}$ be the corresponding state in $p^{\prime}$. Since $c_{j}$ is an initial state and $p^{\prime}$ cannot deadlock, there must be some transition from $c_{j}$. This transition terminates in either $P_{i}$ or $R_{i}$, for some $i$. If the transition from $c_{j}$ terminates in $P_{i}$ then $y_{j}$ contains literal $x_{i}$ and $x_{i}$ is assigned the truth value true. Hence, $y_{j}$ evaluates to true. If the transition from $c_{j}$ terminates in $R_{i}$ then $P_{i}$ should not be reachable. Otherwise, (i) transitions $\left(R_{i}, b_{i}\right),\left(b_{i}, a_{i}\right)$, and $\left(a_{i}, T_{i}\right)$ must be included to ensure that $R \mapsto T$ is satisfied, and (ii) transitions $\left(P_{i}, a_{i}\right),\left(a_{i}, b_{i}\right)$, and $\left(b_{i}, Q_{i}\right)$ must also be included to guarantee that $P \mapsto Q$ is satisfied. Since the inclusion of all six transitions $\left(P_{i}, a_{i}\right),\left(a_{i}, b_{i}\right),\left(b_{i}, Q_{i}\right)$, $\left(R_{i}, b_{i}\right),\left(b_{i}, a_{i}\right)$, and $\left(a_{i}, T_{i}\right)$ causes violation of $P \mapsto Q$ and $R \mapsto T$, it follows that $P_{i}$ must not be reached in any computation of $p^{\prime}$ if $R_{i}$ is reachable. Thus, if $R_{i}$ is reachable then $x_{i}$ will be assigned the truth value false. Since in this case $y_{j}$ contains $\neg x_{i}$, the disjunction $y_{j}$ evaluates to true. Therefore, the assignment 
of values considered above is a satisfying truth assignment for the given 3-SAT formula.

Theorem 5.1 The addition of two leads-to properties to UNITY programs is NP-complete.

Proof. The NP-hardness of adding two leads-to properties follows from the reduction presented in this section. Also, given a solution (in terms of $p^{\prime}$ consisting of $\left.S_{p^{\prime}}, I_{p^{\prime}}, \delta_{p^{\prime}}\right)$ to the instance of the decision problem, one can verify the requirements (1) $S_{p^{\prime}}=S_{p}$, (2) $I_{p^{\prime}}=I_{p}$, , (3) $\delta_{p^{\prime}} \subseteq \delta_{p}$, and (4) $p^{\prime}$ satisfies spec $_{n}$ in polynomial time. Thus, the membership to NP follows. Therefore, the problem of adding two leads-to properties is NP-complete.

\section{Example: Mutual Exclusion}

In this section, we illustrate the role of the algorithm Add_UNITY in deciding about local or comprehensive redesign of a token passing mutual exclusion (ME) program. We use Dijkstra's guarded commands (actions) [4] as the shorthand for representing the set of program transitions. A guarded command $g \rightarrow s t$ captures the transitions $\left\{\left(s_{0}, s_{1}\right)\right.$ : the state predicate $g$ is true in $s_{0}$, and $s_{1}$ is obtained by atomic execution of statement st in state $\left.s_{0}\right\}$.

The initial ME program has two competing processes $P_{1}$ and $P_{2}$. Each process $P_{j}(j=0,1)$ has three Boolean variables $n_{j}, c_{j}$, and $t_{j}$, where (i) $t_{j}$ represents whether or not $P_{j}$ is trying to enter its critical section (i.e., trying section), (ii) $c_{j}$ represents whether or not $P_{j}$ is in its critical section, and (iii) $n_{j}$ represents whether or not $P_{j}$ intends to enter its trying section (i.e., non-trying section). The variables of $P_{j}$ are mutually exclusive; i.e., the condition $\left(t_{j} \Rightarrow\left(\neg n_{j} \wedge\right.\right.$ $\left.\left.\neg c_{j}\right)\right) \wedge\left(n_{j} \Rightarrow\left(\neg t_{j} \wedge \neg c_{j}\right)\right) \wedge\left(c_{j} \Rightarrow\left(\neg n_{j} \wedge \neg t_{j}\right)\right)$ holds. We denote a state of ME by $\left\langle s_{0}, s_{1}\right\rangle$, where $s_{0}$ represents the state of $P_{0}$ and $s_{1}$ represents the state of $P_{1}$. Also, we represent the actions of a process $j(j=0,1)$ as follows:

$$
\begin{array}{llll}
M E 1_{j}: & n_{j} \longrightarrow t_{j}:=\text { true } ; n_{j}:=\text { false } ; \\
M E 2_{j}: & t_{j} \longrightarrow c_{j}:=\text { true } ; t_{j}:=\text { false } \\
M E 3_{j}: & c_{j} \longrightarrow n_{j}:=\text { true } ; c_{j}:=\text { false } ;
\end{array}
$$

For simplicity, we illustrate the reachability graph of the initial ME program in Figure 4 that shows all reachable states from the initial state $s_{\text {init }}$, where both processes are in their non-critical sections. We have annotated each transition with the index of the process that executes that transition.

In the initial state of $\mathrm{ME}$, both processes are in their non-trying section (i.e., $n_{0}=$ true and $n_{1}=$ true). The ME program satisfies its safety property that stipulates $P_{0}$ and $P_{1}$ must not enter the critical section simultaneously (i.e., Invariant $\left.\left(\neg\left(c_{0} \wedge c_{1}\right)\right)\right)$. Also, the initial ME program only satisfies $c_{j} \mapsto n_{j}$. Next, we trace Add_UNITY to add the leads-to property $t_{0} \mapsto c_{0}$ to ME while preserving $c_{0} \mapsto n_{0}$. For reasons of space, we omit the addition of $t_{1} \mapsto c_{1}$ as it is similar to the addition of $t_{0} \mapsto c_{0}$.

Step 1. Since ME already satisfies its safety property, no transitions are removed at the first step of Add_UNITY. 


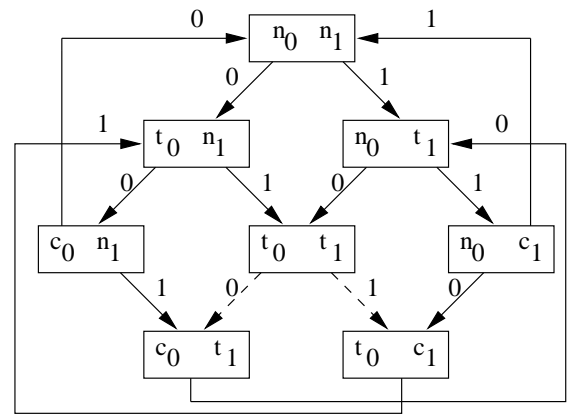

Fig. 4. The reachability graph of program $M E$.

Step 2. The Add_UNITY algorithm ranks all states based on their shortest computation prefix to states where $c_{0}$ is true. As a result, the rank of $\left\langle t_{0}, t_{1}\right\rangle$ becomes 1 and the rank of $\left\langle t_{0}, c_{1}\right\rangle$ becomes 2 .

Step 3. Since there exist no states with rank $\infty$, Add_UNITY does not remove any transitions in Step 3.

Step 4. Since the execution of Steps 2 and 3 does not create any deadlock states, Add_UNITY does not modify the program structure in Step 4.

Step 5 and 6. The ranking of the states will not be changed in Step 5. Also, Add_UNITY exits the repeat-until loop since no state where $t_{0}$ holds has a rank of $\infty$.

Step 7. Finally, in Step 7, Add_UNITY removes the transition $\left\langle t_{0}, t_{1}\right\rangle \rightarrow\left\langle t_{0}, c_{1}\right\rangle$ since the rank of $\left\langle t_{0}, t_{1}\right\rangle$ is 1 and the rank of $\left\langle t_{0}, c_{1}\right\rangle$ is 2 (see Figure 4 ). Likewise, in the addition of $t_{1} \mapsto c_{1}$, the transition $\left\langle t_{0}, t_{1}\right\rangle \rightarrow\left\langle c_{0}, t_{1}\right\rangle$ is removed (see Figure $4)$.

A similar execution of Add_UNITY for the addition of $t_{1} \mapsto c_{1}$ results in the synthesis of the following ( $\oplus$ denotes modulo 2 addition):

$$
\begin{aligned}
& M E 1_{j}^{\prime}: \quad n_{j} \wedge \neg t_{(j \oplus 1)} \longrightarrow t_{j}:=\text { true } ; n_{j}:=\text { false; } \\
& M E 2_{j}^{\prime}: t_{j} \wedge n_{(j \oplus 1)} \longrightarrow c_{j}:=\text { true } ; t_{j}:=\text { false; } \\
& M E 3_{j}^{\prime}: \quad c_{j} \quad \longrightarrow n_{j}:=\text { true } ; c_{j}:=\text { false; }
\end{aligned}
$$

Note that, the above program does not satisfy $n_{j} \mapsto t_{j}$. Now, if we use Add_UNITY for the addition of $n_{j} \mapsto t_{j}$, while preserving $t_{j} \mapsto c_{j}$ and $c_{j} \mapsto n_{j}$, then Add_UNITY will declare failure because the initial state will be deadlocked. In the context of this example, the addition of the above properties will fail regardless of the order of their addition. Thus, based on the completeness of Add_UNITY, it follows that the initial program cannot be revised to a program that simultaneously satisfies the above leads-to properties. This is an interesting result that enlightens designers to search for other solutions where one adds new variables and computations to the ME program (e.g., Peterson's solution) instead of spending time on modifying the initial ME program.

\section{Related Work}

In this section, we illustrate how the contributions of this paper differ from existing approaches for program synthesis and verification. Existing synthesis 
methods in the literature mostly focus on deriving the synchronization skeleton of a program from its specification (expressed in terms of temporal logic expressions or finite-state automata) [5-11], where the synchronization skeleton of a program is an abstract structure of the code of the program implementing inter-process synchronization. Although such synthesis methods may have differences with respect to the input specification language and the program model that they synthesize, the general approach is based on the satisfiability proof of the specification. This makes it difficult to provide reuse in the synthesis of programs; i.e., any change in the specification requires the synthesis to be restarted from scratch. By contrast, since the input to our algorithm (cf. Figure 1) is the set of transitions of a program, our approach has the potential to reuse those transitions in incremental synthesis of a revised version of the input program.

The algorithms for automatic addition of fault-tolerance [12-16] add faulttolerance concerns to existing programs in the presence of faults, and guarantee not to add new behaviors to that program in the absence of faults. The problem of adding fault-tolerance is orthogonal to the problem of adding UNITY properties in that one could use the algorithms of [12-16] to add fault-tolerance concerns to a UNITY program synthesized by the algorithm presented in this paper. On the other hand, we plan to investigate the addition of UNITY properties to fault-tolerant programs while preserving their fault-tolerance properties.

Run-time verification. Runtime verification techniques focus on monitoring the program behavior at runtime with respect to a given specification [17]. Also, such techniques provide a mechanism for ensuring the correctness of program execution after monitoring violations of desired properties [18]. Such approaches mostly focus on the verification of safety properties [19-22] and also provide mechanisms for exception handling and dealing with deadlocks at runtime. By contrast, our focus is on off-line addition of UNITY properties to programs where we ensure that the synthesized program satisfies its existing and newly added properties. Also, to the best of our knowledge, the runtime verification of leads-to properties is still an open question.

\section{Discussion}

In this section, we address some questions raised about the limitations and the applications of the results presented in this paper. We proceed as follows:

Stepwise application of Add_UNITY. The Add_UNITY algorithm can be used in a stepwise fashion. While such a stepwise use of Add_UNITY to add multiple leads-to properties will be sound, it is not complete. This is due to the fact that during the addition of the first leads-to property, the transitions removed in the last step (Step 7 in Figure 1) may cause failure in adding the subsequent leadsto property. Therefore, this does not contradict the NP-completeness result in Section 5.

Addition of other UNITY properties. The Add_UNITY algorithm shows that it is possible to add several safety (stable, invariant and unless) properties and one leads-to property in polynomial time. Since ensures is a conjunction of unless and leads-to properties, this algorithm can be trivially extended to deal 
with the case where one adds several safety properties and an ensures property. Also, one can use Add_UNITY to add the until property in Linear Temporal Logic (LTL) [23] to programs as ensures is semantically the same as until in LTL.

However, in the context of adding multiple leads-to properties, there are several open questions. For example, is it possible to combine these leads-to properties with other (specific) properties to obtain efficient solutions? To illustrate this, it is straightforward to observe that adding 'invariant $(\neg P) \wedge$ $(P \mapsto Q) \wedge(R \mapsto T)^{\prime}$ ' can be added efficiently, as it corresponds to adding 'invariant $(\neg P) \wedge(R \mapsto T)$ '. Moreover, the complexity of adding two ensures properties is still an open question. (Note that the complexity of adding two ensures properties does not necessarily follow from the results in Section 5; as discussed earlier in this paragraph, combining leads-to properties with certain safety properties, does permit polynomial time solutions.)

Implementing Add_UNITY using model checking. The algorithm Add_UNITY can also be implemented with the help of a model checker as follows: For this exposition, consider the case where a program, say $p$, is specified as a set of transitions, as defined in Section 2. When $p$ is checked with a model checker with respect to a leads-to property $(R \mapsto T)$ and found to be incorrect, the counterexamples will be of one of the following two forms: (1) There exists a state $s_{d}$ such that $s_{d}$ is reachable in computations of $p$ and $s_{d}$ is a deadlocked state, or (2) There exists a state, say $s_{r} \in R$ that is reachable in a program computation and that program computation can be extended to reach a cycle, say $s_{0}, s_{1}, \cdots, s_{n}\left(=s_{0}\right)$ such that $T$ is never satisfied. In the former case, transitions terminating in $s_{d}$ need to be removed. In the latter case, we need to check if there exists a computation prefix of $p$ that starts in one of the states in the cycle and reaches $T$. (This case could also be checked with a model checker.) If such a computation prefix does not exist then the state $s_{r}$ and all its incident transitions should be removed. If such a computation prefix exists and $s_{j}$ is the last state from the cycle to appear on that path then the transition $\left(s_{j}, s_{j+1}\right)$ in the cycle should be removed. After removing the transitions in this fashion, we can repeat the process with the new program until a solution is found. (We leave it to the reader to verify that this approach is also sound and complete.)

The choice of the initial program. The algorithm Add_UNITY takes the initial program $p$ and adds a set of UNITY properties to $p$ if possible. The choice of the initial program can affect the result of addition in that if we start with an initial program that is maximal, i.e., has the maximal non-determinism, then the chance of a successful addition is higher. This issue is particularly important for a step-wise application of the Add_UNITY algorithm.

\section{Conclusion and Future Work}

In this paper, we focused on the problem of revising UNITY [1] programs where one adds a conjunction of UNITY properties unless, stable, invariant, ensures, and leads-to to an existing program to provide new functionalities while preserving the existing functionalities. This is an important problem given the dynamic nature of the requirements of computing systems, where developers need to con- 
stantly revise existing programs due to newly-discovered user requirements. In particular, we formally defined the problem of adding UNITY properties to programs. Afterwards, we presented a sound and complete algorithm for such addition where one automatically (i) verifies if it is possible to add a conjunction of UNITY properties to a program and preserve the existing properties, and (ii) adds a conjunction of UNITY properties to a program if such addition is possible.

More importantly, we showed that if one adds a single leads-to property and a conjunction of unless, stable, and invariant properties to a program then the complexity of such addition will be polynomial in program state space. However, in general, we showed a surprising result that simultaneous addition of two leadsto properties to a program is NP-complete. Hence, revising UNITY programs would be significantly easier if one added a single leads-to property instead of adding more than one leads-to property. Since ensures can be expressed as the conjunction of an unless property and a leads-to property, the algorithm presented in this paper for adding a leads-to property and a conjunction of unless, stable, and invariant properties can be used for the addition of ensures property as well. Nonetheless, to the best of our knowledge, the complexity of adding two ensures properties to UNITY programs is still an open problem.

To extend the results of this paper, we plan to integrate the algorithm presented in this paper with model checking algorithms to provide automated assistance for developers. As a result, if the model checking of a model with respect to a UNITY property fails then our algorithm automatically (i) determines whether or not the model is fixable, and (ii) fixes the model if it is fixable.

\section{References}

1. K. M. Chandy and J. Misra. Parallel Program Design: A Foundation. AddisonWesley, 1988.

2. B. Alpern and F. B. Schneider. Defining liveness. Information Processing Letters, 21:181-185, 1985.

3. Ali Ebnenasir and Sandeep Kulkarni. Automatic addition of liveness. Technical Report MSU-CSE-04-22, Department of Computer Science, Michigan State University, East Lansing, Michigan, June 2004.

4. E. W. Dijkstra. A Discipline of Programming. Prentice-Hall, 1990.

5. E.A. Emerson and E.M. Clarke. Using branching time temporal logic to synthesize synchronization skeletons. Science of Computer Programming, 2(3):241-266, 1982.

6. Z. Manna and P. Wolper. Synthesis of communicating processes from temporal logic specifications. ACM Transactions on Programming Languages and Systems, 6(1):68-93, 1984.

7. A. Pnueli and R. Rosner. On the synthesis of a reactive module. In Proceedings of the 16th ACM Symposium on Principles of Programming Languages, pages 179190, 1989.

8. A. Pnueli and R. Rosner. On the synthesis of an asynchronous reactive module. In Proceeding of 16th International Colloqium on Automata, Languages, and Programming, Lec. Notes in Computer Science 372, Springer-Verlag:652-671, 1989. 
9. A. Arora, P. C. Attie, and E. A. Emerson. Synthesis of fault-tolerant concurrent programs. ACM Transactions on Programming Languages and Systems (TOPLAS), 26(1):125-185, 2004. (A preliminary version of this paper appeared in Proceedings of the 17th ACM Symposium on Principles of Distributed Computing, 1998.).

10. P. Attie. Synthesis of large concurrent programs via pairwise composition. $C O N-$ CUR'99: 10th International Conference on Concurrency Theory, Lecture Notes In Computer Science, 1664:130-145, 1999.

11. P. Attie and A. Emerson. Synthesis of concurrent programs for an atomic read/write model of computation. ACM TOPLAS, 23(2):187-242, March 2001. (A preliminary version of this paper appeared in PODC96.).

12. S. S. Kulkarni and A. Arora. Automating the addition of fault-tolerance. Proceedings of the 6th International Symposium of Formal Techniques in Real-Time and Fault-Tolerant Systems, pages 82-93, 2000.

13. S. S. Kulkarni, A. Arora, and A. Chippada. Polynomial time synthesis of Byzantine agreement. Symposium on Reliable Distributed Systems, pages 130-139, 2001.

14. S. S. Kulkarni and A. Ebnenasir. The complexity of adding failsafe fault-tolerance. Proceedings of the 22nd International Conference on Distributed Computing Systems, pages 337-344, 2002.

15. S. S. Kulkarni and A. Ebnenasir. Enhancing the fault-tolerance of nonmasking programs. Proceedings of the 23rd International Conference on Distributed Computing Systems, pages 441-449, 2003.

16. S. S. Kulkarni and A. Ebnenasir. Automated synthesis of multitolerance. In Proceedings of the International Conference on Dependable Systems and Networks, Palazzo dei Congressi, Florence, Italy, pages 209 - 218, June 28 - July 12004.

17. K. Havelund and G. Rosu. Runtime verification. Formal Methods in System Design. Special issue dedicated to $R V^{\prime} 01,24(2), 2004$.

18. F. Chen, M. D'Amorim, and G. Rosu. A formal monitoring-based framework for software development and analysis. Sixth International Conference on Formal Engineering Methods (ICFEM), pages 357-372, November 2004.

19. Bernd Fisher, Johann Schumann, and Mike Whalen. Synthesizing certified code. Proceedings of the International Symposium of Formal Methods Europe (FME'02), Lecture Notes In Computer Science, 2391:431-450, 2002.

20. Ewen Denney, Bernd Fischer, and Johann Schumann. Adding assurance to automatically generated code. In Proceedings the 8th IEEE International Symposium on High Assurance Systems Engineering (HASE 2004), pages 297-299, March 2004.

21. Klaus Havelund and Grigore Rosu. Synthesizing monitors for safety properties. In Tools and Algorithms for Construction and Analysis of Systems (TACAS'02), volume 2280 of Lecture Notes in Computer Science, pages 342-356, October 2002.

22. K. Sen, G. Rosu, and G. Agha. Runtime safety analysis of multithreaded programs. In ACM SIGSOFT Conference on the Foundations of Software Engineering /European Software Engineering Conference, Helsinki, Finland, pages 337-346, 2003.

23. E.A. Emerson. Handbook of Theoretical Computer Science: Chapter 16, Temporal and Modal Logic. Elsevier Science Publishers B. V., 1990. 